\title{
Retrospective cost-effectiveness of the 23-valent pneumococcal polysaccharide vaccination program in Australia
}

\author{
Paul Van Buynder, Allan W. Cripps
}

The manuscript by Chen and colleagues [1] suggests that, based on retrospective Australian data, 23valent pneumococcal polysaccharide vaccine (PPV23) has a worsening cost-effectiveness profile over time and is unlikely to be cost-effective unless it provides protection against non-invasive community acquired pneumococcal pneumonia (pneumococcal CAP). At an effectiveness of about $15 \%$ against pneumococcal CAP, the PPV23 program approached cost-effectiveness [1].

In their base case, Chen and colleagues assumed zero benefit from PPV23 against pneumococcal CAP. Such an assumption is inconsistent with recent studies which suggest PPV23 effectiveness against pneumococcal CAP is non-zero and could be as high as 50\% (reviewed in Van Buynder and Booy [2]). Studies supportive of an effectiveness for PPV23 against vaccine-type (VT) pneumococcal CAP include: a Japanese prospective cohort study which found 33\% VE (95\% CI: 5.6-53.1\%) against VT pneumococcal CAP in patients that had received at least one dose of PPV23 in the 5 years before [3]; a Spanish cohort study which found 48\% (95\% CI: 8-71\%) VE against non-bacteraemic pneumococcal CAP in subjects vaccinated up to 5 years previously [4]; and a German meta-analytical review which allocated a VE against pneumococcal CAP of $64 \%$ (95\% CI: 35-80\%) out to 2.5 years in randomised clinical trials and of 48\% (95\% CI: 25-62\%) in cohort studies followed out to 5 years [5]. Re-examination of the cost-effectiveness of the PPV23 program applying assumptions regarding effectiveness for PPV23 against VT pneumococcal CAP consistent with the literature described above would greatly enhance the contribution of this publication to public health decision making. 
Disease due to Streptococcus pneumoniae remains a major source of illness in older persons and globally it remains the most important pathogen in respiratory infection deaths. Changes to the current universal pneumococcal vaccination program could place older persons at an increased risk of death from a vaccine preventable disease and should not be undertaken lightly. Indeed, many national advisory bodies have recommended continuation of a PPV23 immunisation for older persons.

There are sufficient studies to suggest that PPV23 effectiveness against VT pneumococcal CAP could be as high as $50 \%$ and the duration of effectiveness may stretch to 5 years. Based on the sensitivity analyses presented by Chen and colleagues, the PPV23 program would likely be highly cost-effective under such assumptions. Furthermore, with the increasing burden of disease due to non-pneumococcal conjugate vaccine serotypes, universal immunisation of those aged 65 years and over should be retained in Australia, particularly given the epidemiological data which demonstrates a substantially lower rate of increase in invasive pneumococcal disease attributable to PPV23 serotypes compared to non PPV23 serotypes over the past decade [6].

\section{References}

1. C. Chen, P. Beutels, J. Wood, et al. Retrospective cost-effectiveness of the 23-valent pneumococcal polysaccharide vaccination program in Australia. Vaccine, 36 (2018), pp. 6307-6313

2. P.G. Van Buynder, R. Booy. Pneumococcal vaccination in older persons: where are we today? Pneumonia, 10 (2018), p. 1, 10.1186/s41479-017-0045-y

3. M. Suzuki, Bhim Gopal Dhoubhadel, T. Ishifuji, Michio Yasunami, Makito Yaegashi, Norichika Asoh, Masayuki Ishida, Sugihiro Hamaguchi, Masahiro Aoshima, Koya Ariyoshi, Konosuke Morimoto. Serotype-specific effectiveness of 23-valent pneumococcal polysaccharide vaccine against pneumococcal pneumonia in adults aged 65 years or older: a multicentre, prospective, test-negative design study. Lancet Infect Dis, 17 (3) (2017), pp. 313321, 10.1016/S1473-3099(17)30049-X

4. O. Ochoa-Gondar, A. Vila-Corcoles, T. Rodriguez-Blanco, et al. Effectiveness of the 23valent pneumococcal polysaccharide vaccine against community-acquired pneumonia in the general population aged $\geq 60$ years: 3 years of follow-up in the CAPAMIS study. Clin Infect Dis, 58 (7) (2014), pp. 909-917

5. G. Falkenhorst, C. Remschmidt, T. Harder, E. Hummers-Pradier, G. Wichmann, C. Bogdan. Effectiveness of the 23 valent pneumococcal polysaccharide vaccine (PPV23) against pneumococcal disease in the elderly: systematic review and meta-analysis. PLoS ONE, 12 (1) (2017), p. e.0177985 
6. Stein A, Cripps A, Litt J and Menzies R. Epidemiology of invasive pneumococcal disease in older Australians reflects impact of childhood and adult pneumococcal vaccination programs. Abstract ISPPD-0319, p645-6. International Symposium of Pneumococci and Pneumococcal Disease. 2018; available from isppd.kenes.com/2018/Documents/ISPPD-

$11 \% 20$ Abstract $\% 20$ Book.pdf. 